\title{
Maylis de Kerangal : une écriture au cœur de la mer
}

\section{Bonnet, Dominique}

Université de Huelva, domi@uhu.es

\section{Resumen}

Maylis de Kerangal nació en Toulon y creció en Le Havre. Esta vida portuaria queda muy presente en su obra y desde Corniche Kennedy hasta À ce stade de la nuit pasando por Réparer les vivants el universo marítimo impregna su escritura y el mar fluye por sus novelas. En nuestro artículo intentaremos demostrar el papel preponderante que desempeña el mar en la creación literaria de Maylis de Kerangal dejándonos llevar por su imaginario marítimo : "Je me dis parfois qu'écrire c'est instaurer un paysage. Les îles, et plus encore les îles désertes, sont pour cela des matériaux de haute volée, leur statut géologique amorçant déjà une écriture, portant un récit. Essaimées sur la mer, les îles surgissent comme des creusets à fictions, ou des aimants dispersés sur l’imaginaire » (À ce stade de la nuit, 2015).

Palabras clave : Maylis de Kerangal ; islas ; orilla ; fronteras ; paisaje.

\section{Résumé}

Maylis de Kerangal est née à Toulon et a grandi au Havre. Cette vie portuaire reste très présente dans son œuvre et de Corniche Kennedy à À ce stade de la nuit en passant par Réparer les vivants l'univers maritime marque son écriture et la mer traverse ses romans. Dans notre article nous essaierons de montrer le rôle prépondérant que joue la mer dans la création littéraire de Maylis de Kerangal tout en nous laissant porter par sa conception de l'écriture tant imprégnée par l'imaginaire maritime : "Je me dis parfois qu'écrire c'est instaurer un paysage. Les îles, et plus encore les îles désertes, sont pour cela des matériaux de haute volée, leur statut géologique amorçant déjà une écriture, portant un récit. Essaimées sur la mer, les îles surgissent comme des creusets à fictions, ou des aimants dispersés sur l'imaginaire » (À ce stade de la nuit, 2015).

Mots-clés : Maylis de Kerangal ; îles ; rivage ; frontières ; paysage.

\begin{abstract}
Maylis de Kerangal was born in Toulon and grew up in Le Havre. His life in these harbour towns is very well reflected in novels such as Corniche Kennedy, éparer les vivants, or À ce stade de la nuit, all of them pervaded with powerful sea images. This paper focuses on the key role and meaning of sea imagery in De Kerangal's œuvre: "Je me dis parfois qu'écrire c'est instaurer un paysage. Les îles, et plus encore les îles désertes, sont pour cela des matériaux de haute volée, leur statut géologique amorçant déjà une écriture, portant un récit. Essaimées sur la mer, les îles surgissent comme des creusets à fictions, ou des aimants dispersés sur l’imaginaire » (À ce stade de la nuit, 2015).
\end{abstract}

Keywords : Maylis de Kerangal ; islands ; seashore ; borders ; landscape. 


\title{
Introduction
}

Maylis de Kerangal, femme de lettres, éditrice et romancière, est née à Toulon en 1967 puis a grandi au Havre en Haute Normandie. Dans ces deux villes portuaires aux paysages maritimes différents, Maylis de Kerangal s'imprègne du paysage du rivage, motif très présent dans son œuvre. Son père, sur les traces de son grand-père, a été officier de la marine marchande et capitaine au long cours puis finalement pilote au port du Havre. Sa mère, elle, fut professeur d'histoire géographie, profession qui aura aussi une grande importance dans l'écriture et la vision du monde de Maylis de Kerangal. Cette enfance se retrouve en partie dans son premier roman Je marche sous un ciel de traîne et plus particulièrement dans l'enfance du personnage du libraire qui est rythmée par l'attente puis par les retrouvailles du père après de longs mois en mer. Pour Maylis de Kerangal, le souvenir est fondamental dans l'écriture puisqu'il agit comme un détonateur: «les souvenirs sont des empreintes que l'écriture réactive ${ }^{1}$. L'écriture est une sorte de voyage qui passe par les sens dans la tradition baudelairienne. Cette expérience physique donne lieu à l'élaboration d'un paysage lié à la mémoire et c’est ainsi que pour Maylis de Kerangal écrire c'est instaurer à nouveau un paysage :

\begin{abstract}
Je me dis parfois qu'écrire c’est instaurer un paysage. Les îles, et plus encore les îles désertes, sont pour cela des matériaux de haute volée, leur statut géologique amorçant déjà une écriture, portant un récit. Essaimées sur la mer, les îles surgissent comme des creusets à fictions, ou des aimants dispersés sur l’imaginaire. Elles émergent soudain, formes finies au milieu de l'infini, formes dont on peut saisir les contours et que l'on peut tenir dans un seul geste, comme on tient un caillou dans son poing, comme on cadre une image dans l'objectif de l'appareil , c'est un espace clair qui impose ses contours, créant aussitôt un dedans et un dehors : les îles sont comme des idées [...] On y échoue par le chant des sirènes, à l'issue d'une tempête qui aura éclaté la coque du navire, après s'être évadé d’un bâtiment ennemi [...] on aura nagé pendant des heures, flotté parfois plusieurs jours accroché à une poutre, ou finalement lâché prise, et d'épuisement confié son corps à la vague [...] plus tard on s’y réveille, on y survit, on est comme dans un rêve, parfois on y recrée le monde, le feu et la forge, la chasse et la cueillette, on y repense la politique, le régime de pouvoir et de propriété Kerangal, 2015a : 56-57).
\end{abstract}

Dans cette notion de paysage sa condition de fille et de petite-fille de marins au long cours l'a accoutumée à la mer mais aussi aux îles et donc au rivage. Son rapport à la mer passe donc par la carte maritime afin d'y situer les îles, d'en calculer leur distance, la mer devenant de la sorte un espace géopolitique ${ }^{2}$. C'est ce rapport à la mer qui nous intéresse et que nous allons tenter de percevoir dans trois livres de Maylis de Kerangal que nous étudierons dans la chronologie de leur écriture : Corniche Kennedy, Réparer les vivants et pour terminer À ce stade de la nuit.

\section{La mer comme lisière dans Corniche Kennedy}

Corniche Kennedy, publié en 2008, est le troisième roman de Maylis de Kerangal. L’auteur nous plonge (au sens réel et littéraire...) au sein d'un groupe d'adolescents qui passe l'été sur une dalle au bord de la mer à Marseille et qui se met en danger en sautant dans la mer, de rochers de plus en plus haut afin d'échapper à la solitude étouffante de leurs appartements, de leurs cités, de leurs maisons, de leur ennui. En plongeant, ils «se précipitent alors dans le ciel, dans la mer, dans toutes les profondeurs possibles, et quand ils sont dans l'air, hurlent ensemble, un même cri, accueillis soudain plus vivants et plus vastes dans un plus vaste monde » (Kerangal, 2015b : 39). Dès le début du livre trois d'entre eux, Eddy, Suzanne et Mario se détachent du reste des jeunes. Tous les trois proviennent de milieux différents : Eddy vit dans une banlieue résidentielle pour classes moyennes et c'est le chef de la bande, Mario encore très jeune est livré à lui-même dans un HLM et Suzanne issue d'un milieu aisé habite une villa sur la corniche. Ils sont unis parce qu'ils bravent l'interdit en sautant et que cette exaltation leur permet d'échapper au monde ne serait-ce qu'un instant. De l'autre côté de la Corniche, dans son bureau de police, Sylvestre Opéra les observe et les surveille entre fascination et horreur. Sauter est hallucinant comme l'affirme Eddy (Kerangal, 2015b : 39) mais par un décret du Maire de la ville ces plongeons deviennent interdits de par le danger qu’ils représentent. Sylvestre Opéra, incarnation de l'autorité doit alors tenter de stopper ces défis en arrêtant tous ces jeunes malgré d'autres affaires beaucoup plus graves qui

\footnotetext{
${ }^{1}$ Interview de Maylis de Kerangal, La Grande Librairie, https://www.youtube.com/watch?v=3HPsexWCpJM, écouté le 3 mars 2016.

${ }^{2}$ Idem.
} 
l'occupent: trafic de drogues, prostitution, mafia, meurtre... Dans ce climat de pression politique c'est une véritable lutte qui s'engage entre la bande de la Plate et la police.

Dans Corniche Kennedy la mer qui sépare la plate (roche plate et lisse sur laquelle se retrouvent les jeunes) du reste du monde constitue le lien qui mène au récit, au double récit de ces deux mondes :

\begin{abstract}
Ils se donnent rendez-vous au sortir du virage, après Malmousque, quand la corniche réapparaît au-dessus du littoral, voie rapide frayée entre terre et mer, lisière d'asphalte. Longue et mince, elle épouse la côte tout autant qu'elle contient la ville, en ceinture les excès, congestionnée aux heures de pointe, fluide la nuit - et lumineuse alors, son tracé fluorescent sinue dans les focales des satellites placés en orbite dans la stratosphère. Elle joue comme un seuil magnétique à la marge du continent, zone de contact et non frontière, puisqu'on la sait poreuse, percée de passages et d'escaliers qui montent vers les vieux quartiers, ou descendent sur les rochers. L'observant, on pense à un front déployé que la vie affecte de tous côtés, une ligne de fuite, planétaire, sans extrémités : on y est toujours au milieu de quelque chose, en plein dedans. C’est là que ça se passe et c’est là que nous somme (Kerangal, 2015b : 9).
\end{abstract}

La mer c'est aussi le lien entre Tania et ce monde, cette prostituée migrante échouée sur la Corniche Kennedy qui se chauffe enfin doucement au soleil, après être arrivée par cette mer qui s'étend maintenant sous ses yeux, elle qui, presque miraculeusement, est vivante vivante :

[...] elle (Tania) s'était retrouvée un dimanche d'avril sur la corniche Kennedy, assise sur le parapet de béton, paupières closes chauffées doucement sous le soleil tendre, la mer bruissant au-devant d'elle, échouée mais vivante, échouée mais vivante, vivante vivante vivante, un moment de grâce qui n’a pas duré (Kerangal, 2015b : 76)

L’adjectif vivante répété tel le mouvement des vagues, tel le ressac, exalte le lieu évoqué par Tania tout en renforçant la symbiose entre paysage et personnage.

Comme nous l'explique Judith Josse-Laffon (2015 : 199) le roman se construit dans une tension entre deux lieux : le commissariat, espace clos s'oppose à la Corniche, espace ouvert vers l'extérieur. L'espace clos est l'univers des adultes mais c'est aussi le territoire de la ville, le paysage urbain. La Corniche, en revanche, constitue l'espace ouvert, solaire, minéral occupée par la jeunesse :

Il a parlé dans un souffle, depuis quelques secondes, la fille resplendit sous le soleil horizontal, ciblée en pleine tête comme le naos au fond du temple, et sa peau s'est dorée d'un coup, peau d'héritière, lisse et douce, irisée d'ambre solaire, pieds bronzés, ongles nacrés, un paréo tahitien, trois glaçons dans un verre à orangeade [...] Eddy trouve qu'il n'y a rien de plus passionnant à cette minute que cette peau de fille, là, toute concrète, membrane qui palpite, absorbe et transmet, tissu qui capte et décongestionne, rien de plus troublant que cette peau (Kerangal, 2015b : 37)

L'espace clos de Sylvestre Opéra s'oppose alors à celui de la fiction en mouvement qui évolue au rythme du mouvement de la jeunesse. La Corniche reste ainsi la frontière entre ces deux mondes et elle constitue le point de départ de l'inspiration de Kerangal, la lisière, la côte, le lieu de rencontre entre terre et mer. Elle ouvre et ferme le livre et s’offre à nous comme l'espace de renaissance de l'ensemble des personnages :

Ils longent calmement l'aplomb de la gorge et suivent la ligne de crête jusqu’à la mer la plus claire [...] Ils sont face à l'abîme, des herbes ont poussé sur l'arête de la falaise, ultime rempart végétal avant le puits cobalt du ciel [...] Eddy dénoue sa ceinture et détache le paquet, soupèse, bras tendu. Ensemble, ils posent les yeux sur l'entaille blanche comme s'ils touchaient une boîte magique, puis le garçon élève le paquet dans le soleil pour faire reluire le plastique [...] Le colis miroite comme une source (Kerangal, 2015b : 149) 


\section{La puissance organique et créatrice de la vague dans Réparer les vivants}

Réparer les vivants, publié en 2014, emprunte son titre à Anton Tchekov qui fut, comme le souligne Maylis de Kerangal au cours d'une interview ${ }^{3}$, médecin, malade (emporté par la tuberculose), et bien sûr écrivain. Dans la pièce Platonov, un des personnages affirme qu'il ne reste plus qu'à " enterrer les morts et réparer les vivants ». C’est cette phrase, qu'à son tour un des personnages de Maylis de Kerangal accroche sur sa porte. Il s'agit de l'infirmier Thomas Réminge, coordonnateur de greffe, dont le rôle est de proposer aux parents des victimes le don d'organe puis d'initier et d'accompagner tout le processus chirurgical, jusqu’à la toilette mortuaire. Il représente en quelque sorte l'intermédiaire entre la mort et la vie nouvelle. Réparer les vivants est le récit d'une greffe qui se joue en l'espace de vingt-quatre heures. Après un accident qui plonge Simon Limbres dans un coma irréversible, son cœur va devenir celui de Claire Méjan.

Dans Réparer les vivants, la mer est présente sous la forme d’une vague, la vague qui indirectement causera la perte du très jeune Simon Limbres ${ }^{4}$ mais qui de façon paradoxale aussi le maintiendra dans le monde de la vie de façon presque éternelle.

La vague, motif très fort, incarne la force organique. Elle ouvre et ferme l’idée du cycle éternel tout en représentant un espace incessant, porteuse du flux d’énergie. Dans une interview Maylis de Kerangal affirme avoir écrit Réparer les vivants en continu tout comme le surf suit le mouvement de la vague ${ }^{5}$, la scène inaugurale donnant tout son sens au récit en amorçant cette écriture du déferlement :

C’est l'heure. Amorce du jour où l'informe prend forme : les éléments s'organisent, le ciel se sépare de la mer, l'horizon se discerne [...] ils entrent dans l'eau. Ne hurlent pas en y plongeant leurs corps, moulé de cette membrane flexible qui conserve la chaleur des chairs et l'explosivité des élans, n'émettent pas un cri, mais traversent en grimaçant la muraille de cailloux qui roulent, et la mer se creusant vite, puisqu'à cinq ou six mètres du bord ils n’ont déjà plus pied, ils basculent en avant, s'allongent à plat ventre sur leur planche, leurs bras entaillant le flot avec force, ils franchissent la zone de ressac et progressent vers le large [...] À deux cents mètres du rivage, la mer n'est plus qu'une tension ondulatoire, elle se creuse et se bombe, soulevée comme un drap lancé sur un sommier. Simon Limbres se fond dans son mouvement [...] L'eau est sombre, marbrée, veineuse, la couleur de l'étain (Kerangal, $2014: 20)$

Cette scène initiale est celle où Simon Limbres se lève à l'aube pour aller surfer avec ses amis. En sortant de l'eau, épuisés ils auront un accident sur la route du retour. Cette scène initiale telle une vague se déferle au début du roman et ne se refermera qu’à la fin lorsque le cœur de Simon Limbres rebattra dans un autre corps: «Alors, le cœur se contracte, un tressaillement puis ce sont des secousses quasi imperceptibles [...] et l'organe peu à peu commence à pomper le sang dans le corps » (Kerangal, 2014 : 298). Ainsi «Au-delà de l'accident lui-même, c’est donc la description première de la mer et la métaphore filée du surf comme sensation primitive et communion avec la nature, qui importe ${ }^{6}$.

Malgré l'état de mort cérébrale de Simon Limbres après son accident de circulation, le récit de Maylis de Kerangal bouillonne de vie. Simon Limbres restera même après sa mort du côté de cette vie, la force de la vague continuant de le porter au sein de cet univers de jeunesse et de beauté :

Le corps de Simon Limbres apparaît dans la lumière, plus nu que nu soudain [...] Thomas lave le corps, ses mouvements sont calmes et déliés [...] il reconstruit la singularité de Simon Limbres. Il fait surgir le jeune homme de

\footnotetext{
${ }^{3}$ Interview de Maylis de Kerangal sur https://www.youtube.com/watch?v=4uLu6Cx97GM, Sauramps Librairies, écouté le 3 mars 2016.

${ }^{4}$ Maylis de Kerangal explique le nom de Simon Limbre par un mélange entre les Limbes du Moyen Âge où les enfants partaient avant d’être baptisé et le $R$ de l'ombre, de la mort, de l'obscurité de la mer dans la nuit, de la mort froide dans la nuit (Interview de Maylis de Kerangal sur https://www.youtube.com/watch?v=4uLu6Cx97GM, Sauramps Librairies, écouté le 3 mars 2016).

${ }^{5}$ Interview de Maylis de Kerangal sur https://www.youtube.com/watch?v=4uLu6Cx97GM, Sauramps Librairies, écouté le 3 mars 2016.

${ }^{6}$ https://lesecritsvains.com/2014/03/19/reparer-les-vivants-maylis-de-kerangal, consulté le 3 mars 2016.
} 
la dune un surf sous le bras, il le fait courir au-devant du rivage avec d'autres que lui [...] il le propulse dans un espace post mortem que la mort n'atteint plus, celui de la gloire immortelle, celui des mythographies, celui du chant et de l'écriture (Kerangal, $2014: 288$ ).

La narration maintient un équilibre permanent, celui du surfer sur la vague, entre science et philosophie. La donation d’organes passe au second plan et le récit va fouiller dans la mémoire du cœur, dans celle des sensations et des perceptions perçues par Simon Limbres lorsque «l'espace l'envahit, l’écrase tout autant qu'il le libère, sature ses fibres musculaires, ses bronches, oxygène son sang ; la vague se déplie dans une temporalité trouble, lente ou rapide on ne sait pas, elle suspend chaque seconde une à une jusqu’à finir pulvérisée » (Kerangal, 2014 : 23).

\title{
3. À ce stade de la nuit ou le ressac engagé de Maylis de Kerangal
}

Ce motif de la vague nous le retrouvons dans le dernier récit de Maylis de Kerangal, À ce stade de la nuit, sous la forme d'une onde, onde de choc qui provoque le déplacement des idées qui font que les digressions forment le récit ${ }^{7}$. C’est à partir du nom de Lampedusa que se cristallise le récit provoqué par la méditation de Kerangal. Ce nom propre en convoque d'autres porteurs de références culturelles autour de la mer. Dans une interview Maylis de Kerangal fait allusion au ressac de la mer pour définir ces associations d’idées progressives ${ }^{8}$ tel le mouvement des vagues sur le rivage.

Dans cette même interview elle évoque la première image qui lui vient à l'esprit en écoutant le nom du lieu de la tragédie du naufrage à Lampedusa : c'est le visage de Burt Lancaster dans Le Guépard. Pour elle se tisse alors l'histoire d'un double naufrage en parallèle dans son esprit, celui des migrants mais aussi celui d'une société qui s'engloutit :

Giuseppe Tomasi di Lampedusa est l'auteur d’un seul roman, Le Guépard, publié à titre posthume, en 1958 - ce livre devenu ce film mythique. Ce que l'on peut savoir de lui tient à la description ample et précise qu'il donne de l'aristocratie sicilienne à travers celle de la famille du prince Salina [...] Autobiographie d'une famille, ce roman saisit le «moment » d'un homme et des siens, tisse ensemble livre politique, fresque sociale et méditation sur le temps. Il est porté par le regard du prince Salina [...] Je tremble de plaisir et frotte mes paumes l'une contre l'autre quand je me souviens que Salina est aussi un toponyme, désigne aussi une île de la Méditerranée, celle-ci n pas située au sud de la Sicile comme Lampedusa, mais au nord, dans un autre archipel, celui des îles éoliennes : deux noms pour deux îles. D’un nom à l'autre, la migration se poursuit (Kerangal, 2015a : 31-33).

Dans son ressac personnel, elle repense aussi au film The swimmer dans lequel de nouveau Burt Lancaster qui incarne cette fois Ned Merrill, essaie de survivre dans une société superficielle et surfaite :

\begin{abstract}
Tentative de fuite pour se libérer d'un monde et se réinventer, ou tentative pour rentrer chez soi et retourner à la vie d'avant, désir purificateur de renaître, neuf et vierge, afin de repartir de zéro : l'homme nage jusqu’à épuisement de son projet fou. Burt Lancaster l'incarne en migrant abîmé dans une trajectoire de plus en plus douloureuse, un parcours où son corps fatigue, souffre et se détériore à mesure que croît la sensation d'être étranger dans un monde qui l'entoure, et doutant de sa réalité. Peu à peu, le prince Salina et Ned Merrill m’apparaissent comme deux versions d'une même humanité, le recto et le verso d’un même homme [...] le prince et le migrant (Kerangal, 2015a : 16-17).
\end{abstract}

Par ce flux d'idées à partir de ce dramatique naufrage face à l'île de Lampedusa Maylis de Kerangal nous embarque dans une sinuosité d’images disséminées dans l’espace méditerranéen. Tout comme dans le film de Visconti, Maylis de Kerangal ressent la très grande violence de la fin d'un règne : «J'ai réalisé que Visconti avait filmé le bal du Guépard exactement comme un naufrage » (Kerangal, 2015a: 27). Elle affirme que cette histoire très méditerranéenne est

\footnotetext{
${ }^{7}$ http://www.franceculture.fr/emissions/un-autre-jour-est-possible, consultée le 3 mars 2016.

${ }^{8}$ Idem.
} 
l'éternelle histoire des migrations. La Méditerranée comme creuset de toutes les migrations celle de l'Éneïde de Virgile mais aussi celle du voyage d'Ulysse est l'histoire de passage, du transit d'un bord à l'autre. C'est bien sûr l'histoire d'une fuite mais aussi celle de l'hospitalité ${ }^{9}$. Ce récit que compose Maylis de Kerangal s'inscrit dans le cadre d'une commande par la fondation FACIM (Fondation pour l'action culturelle internationale en montagne) pour une collection intitulée Paysages écrits. Cette commande qui dans un premier temps pourrait paraître contraignante devient au contraire une façon d'inscrire des noms dans les paysages. À sa façon Maylis de Kerangal signe ici un récit engagé qui met le doigt sur un monde qui sombre, sur la tragique fermeture des frontières maritimes :

\begin{abstract}
J'ai su que Lampedusa était le nom d'une île il y a une vingtaine d'années, lors des premières arrivées des migrants dans son port et des premiers naufrages dans la zone. À l'époque, ce nom était pour moi celui de Burt Lancaster, celui d'un prince, celui d'un monde qui sombre, celui d'un écrivain, celui du mois d'août, celui d'un enfant. Il feuilletait en désordre différentes couches de sens, activait des imaginaires disparates, instaurait des scènes discontinues, des écritures qui toutes tremblaient dans l'épaisseur de son spectre. Étrangement le toponyme insulaire n’avait encore jamais recouvert le nom de fiction qui avait fini par sédimenter en moi -ce nom de légende, ce nom de cinéma-, mais ce matin, matin du 3 octobre 2013, il s'est retourné comme un gant, Lampedusa concentrant en lui seul la honte et la révolte, le chagrin, désignant désormais un état du monde, un tout autre récit (Kerangal, 2015a : 73).
\end{abstract}

\title{
Conclusion
}

De la plate de Corniche Kennedy au naufrage des migrants de l'île de Lampedusa, Maylis de Kerangal nous porte sur sa vague, mais aussi sur celle du jeune surfer de Réparer les vivants, Simon Limbres, et nous fait toucher les lisières des mondes maritimes qui nous entourent. Sous la plume de Maylis de Kerangal ces rivages délimitent et définissent nos sociétés mais aussi nos sentiments. Cette écriture de la mer qui inonde la narration de Maylis de Kerangal nous maintient en équilibre et nous fait flotter entre deux univers, entre intérieur et extérieur, nous ouvrant l'espace vital et solaire de la vie, de la jeunesse, de la liberté : «À l'origine d’un roman, j’ai toujours des désirs très physiques, matériels. Et une envie d'espaces. Tant qu’il n’y a pas les espaces, il n’y a pas de livre possible » ${ }^{10}$. Une poétique de l’espace maritime dont Maylis de Kerangal fait usage pour explorer le réel...

\section{Références bibliographiques}

Foliot, Michelle (2014). Tisseurs de mots: ateliers d'écriture en Auvergne et ailleurs. <http://www.tisseursdemots.org/spip.php?article543> [Consulté le 3 mars 2016] [personnelle].

France Culture. Un autre jour est possible (12 novembre 2015), <http://www.franceculture.fr/emissions/un-autre-jour-estpossible> [Consultée le 3 mars 2016] [institutionnelle].

Josse-Lafon, Judith (2015). « Dossier » dans Corniche Kennedy, Paris : Folioplus classique, 153-235.

Kerangal, Maylis (2014). Réparer les vivants. Paris : Folio.

Kerangal, Maylis (2015a). À ce stade de la nuit. Paris : Verticales.

Kerangal, Maylis (2015b). Corniche Kennedy. Paris : Folioplus classiques.

Les Écrits Vains. <https://lesecritsvains.com/2014/03/19/reparer-les-vivants-maylis-de-kerangal> [Consultée le 3 mars 2016] [personnelle].

\footnotetext{
${ }^{9}$ Idem.

${ }^{10}$ Interview de Maylis de Kerangal, http://www.tisseursdemots.org/spip.php?article543, consulté le 3 mars 2016.
} 